\title{
Stable classical groups and strongly torsion generated groups
}

\author{
A. J. Berrick and M. Matthey*
}

\begin{abstract}
Strongly torsion generated groups are those with a single normal generator, of arbitrary finite order. They are useful for realizing sequences of abelian groups as homology groups. Known examples include stable alternating groups and stable groups generated by elementary matrices. Here the class of such groups is extended, by consideration of other stable classical groups, including orthogonal and symplectic groups. Discussion of other "classical" groups includes a similar result for the stable special automorphism group of a free group. Failure of such a result for mapping class and braid groups is analyzed. It is also shown that the product of finitely many strongly torsion generated groups is strongly torsion generated.
\end{abstract}

Mathematics Subject Classification (2000). Primary 20F05, 20F28, 20F36, 20G99; Secondary 11E57, 22F50, 37E30.

Keywords. Braid group, free group automorphisms, integral orthogonal group, integral symplectic group, mapping class group, stable general linear group, Steinberg group, strongly torsion generated group.

\section{Introduction}

For $n \geq 2$, a group $G$ is strongly $n$-torsion generated if there is an element $g_{n} \in G$ of order $n$ such that the conjugates of $g_{n}$ generate $G$; that is, the normal closure of $g_{n}$ is all of $G$. A group $G$ is strongly torsion generated if it is strongly $n$-torsion generated for every $n \geq 2$ [2], [7]. Surprisingly, such groups are sufficiently common to generate all possible sequences of homology groups of perfect groups [7]. The constructions of [7] are in the realm of combinatorial group theory; this poses the question of finding "natural" examples of strongly torsion generated groups, which we address here.

For example, since any finite simple group $G$ is strongly $p$-torsion generated for every prime $p$ dividing the order of $G$, the stable alternating group $A_{\infty}$ of even finitary permutations of a countable set is strongly torsion generated [2]; for, $A_{\infty}$ is the direct limit of the alternating groups $A_{k}$.

*2 April 1971 - 15 August 2005

Research partially supported by NUS Research Grant R-146-000-049-112. 
Other historically important examples of strongly torsion generated groups include the subgroup $E(R)$ of the stable general linear group $\mathrm{GL}(R)=\operatorname{dirlim} \mathrm{GL}_{k}(R)$ generated by all elementary matrices and the Steinberg groups $\operatorname{St}(R)$, where $R$ is an associative ring with 1 . These examples featured in the homology and $K$-theory realization results of [2], [5], [6]. The historic examples typically arise from a sequence of like groups $G_{k}$ endowed with natural homomorphisms $G_{k} \rightarrow G_{k+1}$ permitting stabilization (that often respects the formation of homology groups, among other properties) and the construction of the stable group $\underset{\lim }{\longrightarrow} G_{k}$.

Now, stable alternating and general linear groups form part of a web of stable "classical" linear and geometric groups that also involves braid and mapping class groups, automorphism groups of free groups, and orthogonal and symplectic groups (related as in the diagrams of [4], §1). They have topological features in common too (see Remark 2.5 below). This all suggests that study of these other objects might yield further examples. We explore each of these classes below - indeed, because of recent work on torsion in these much-studied groups, new examples do result.

For automorphism groups of free groups and linear groups, the arguments proceed smoothly. There are well-known stabilizations (see Sections 2 and 3 for the definitions), about which we obtain the following results.

Theorem 2.4. The special stable automorphism group $\operatorname{SAut}\left(F_{\infty}\right)$ of the countably infinite free group, and the special stable outer automorphism group $\operatorname{SOut}\left(F_{\infty}\right)$, are strongly torsion generated.

Theorem 3.2. For every form ring $(R, \Lambda)$, every perfect central extension of the perfect commutator subgroup $\mathrm{EU}(R, \Lambda)$ of $U(R, \Lambda)$ is strongly torsion generated.

On the other hand, our analysis reveals a breakdown of the usual analogy between automorphism groups of free groups and mapping class groups. Whereas the former stabilize to yield a strongly torsion generated group, in the case of mapping class groups we observe two contrasting phenomena. Certain mapping class groups $\Gamma_{g, 1}$ are well-known to stabilize with respect to increasing genus $g$ (and, as with the classes above, the stability is respected by the passage to homology groups); however, such mapping class groups are torsion-free and therefore are not amenable to strong torsion generation. For the mapping class groups $\Gamma_{g}$ that do contain torsion, we note some unstable instances of strong $p$-torsion generators. By combining these with known results on the existence of prime torsion in relation to genus, we deduce rigidity results that forbid stabilization. These take the following form.

Corollary 4.6. Let $m \geq 1$, and let $p$ be the largest prime factor of $g(g-1)(2 g+1)$. If

$$
p-\sqrt{2 g}>2 m+2
$$

(and $m \geq 2$ if $p \mid g$ ), then the only homomorphism $\Gamma_{g} \rightarrow \Gamma_{g+m}$ is the trivial homomorphism. 
Similar considerations apply to braid groups. In the cases of torsion, namely the braid groups of the sphere and projective plane, there are vestigial, unstable, results pertaining to strongly $n$-torsion generated groups. Because $n$ depends on the number of strands, these results on strong torsion generators lead to rigidity results that preclude the possibility of stabilization. Hence, as with mapping class groups, strongly torsion generated groups cannot arise, because where stable groups exist, they must be torsion-free.

A final section shows that the product of (a finite number of) strongly torsion generated groups is also strongly torsion generated, a fact applied in [6].

Acknowledgements. The first author benefitted from the hospitality of FIM of ETH, Zürich during the preparation of this paper, and is also grateful to Guido Mislin for stimulating conversations there. He would also like to thank Miguel Xicotencatl and Rocio González for discussions at the 2007 NUS Braids program, and numbertheorists Heng-Huat Chan, Andrew Granville and Greg Martin for remarks in relation to Question 4.7 below. Refereeing comments have also contributed positively to the exposition.

\section{Automorphism groups of free groups}

For $n=1,2, \ldots$, write $F_{n}$ for the free group of rank $n$, with automorphism group abbreviated to $\operatorname{Aut}\left(F_{n}\right)$. The action of $\operatorname{Aut}\left(F_{n}\right)$ on the abelianization of $F_{n}$ may be regarded as a homomorphism from $\operatorname{Aut}\left(F_{n}\right)$ to $\mathrm{GL}_{n}(\mathbb{Z})$ that factors through the outer automorphism group $\operatorname{Out}\left(F_{n}\right)$. The inverse images of the special linear group $\mathrm{SL}_{n}(\mathbb{Z})$ under these maps are normal subgroups denoted here by the special automorphism group $\operatorname{SAut}\left(F_{n}\right)$ and $\operatorname{SOut}\left(F_{n}\right)$ respectively. (Other notations for $\operatorname{SAut}\left(F_{n}\right)$ in the literature include $\mathrm{Aut}^{+}\left(F_{n}\right)$, although that notation invites confusion when the plusconstruction is applied to classifying spaces; and $S A_{n}$, although here we work with $A_{n}$ too.) We thus have the following diagram of pullbacks and vertical inclusions.

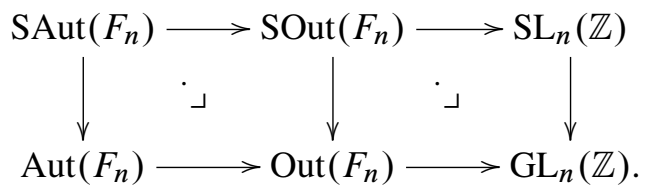

Because inner automorphisms of $F_{n}$ are special, the canonical inclusion $\operatorname{Aut}\left(F_{n}\right) \hookrightarrow$ $\operatorname{Aut}\left(F_{n+1}\right)$, given by trivial action on the final generator of $F_{n+1}$, induces inclusions $\operatorname{SAut}\left(F_{n}\right) \hookrightarrow \operatorname{SAut}\left(F_{n+1}\right)$ and $\operatorname{SOut}\left(F_{n}\right) \hookrightarrow \operatorname{SOut}\left(F_{n+1}\right)$. The colimits of these sequences of inclusions are called $\operatorname{Aut}\left(F_{\infty}\right), \operatorname{SAut}\left(F_{\infty}\right)$ and $\operatorname{SOut}\left(F_{\infty}\right)$, the stable (or finitary, or finite type) automorphism groups of the free group on a countably infinite set of generators. 
Lemma 2.1. For $n \geq 3, \operatorname{SAut}\left(F_{n}\right)$ is the commutator subgroup of $\operatorname{Aut}\left(F_{n}\right)$, and $\operatorname{SOut}\left(F_{n}\right)$ is the commutator subgroup of $\operatorname{Out}\left(F_{n}\right)$.

Proof. By construction, $\operatorname{SAut}\left(F_{n}\right)$ is normal in $\operatorname{Aut}\left(F_{n}\right)$, and

$$
\operatorname{Aut}\left(F_{n}\right) / \operatorname{SAut}\left(F_{n}\right) \cong \operatorname{Out}\left(F_{n}\right) / \operatorname{SOut}\left(F_{n}\right) \cong \operatorname{GL}_{n}(\mathbb{Z}) / \operatorname{SL}_{n}(\mathbb{Z}) \cong C_{2} .
$$

Therefore $\operatorname{Aut}\left(F_{n}\right)_{\mathrm{ab}}$ and $\operatorname{Out}\left(F_{n}\right)_{\mathrm{ab}}$ contain at least two elements.

In the other direction, recall that the symmetric group $\Sigma_{n}$ embeds in $\operatorname{Aut}\left(F_{n}\right)$ by permuting the generators of the free group. From [8], Corollary $1, \operatorname{Aut}\left(F_{n}\right)$ is the normal closure of $\Sigma_{n}$, whence $\left(\Sigma_{n}\right)_{\mathrm{ab}} \cong C_{2}$ maps onto $\operatorname{Aut}\left(F_{n}\right)_{\mathrm{ab}}$.

We conclude that the abelianization of $\operatorname{Aut}\left(F_{n}\right)$ has order 2, making $\operatorname{SAut}\left(F_{n}\right)$ the commutator subgroup of $\operatorname{Aut}\left(F_{n}\right)$. The epimorphism from $\operatorname{Aut}\left(F_{n}\right)$ to $\operatorname{Out}\left(F_{n}\right)$ induces an epimorphism of their abelianizations. Thus also $\operatorname{Out}\left(F_{n}\right)_{\mathrm{ab}} \cong C_{2}$, and $\operatorname{SOut}\left(F_{n}\right)$ is the commutator subgroup of $\operatorname{Out}\left(F_{n}\right)$. In effect, this is the same phenomenon as $A_{n}$ and $\mathrm{SL}_{n}(\mathbb{Z})(n \geq 3)$ being the commutator subgroups of $\Sigma_{n}$ and $\mathrm{GL}_{n}(\mathbb{Z})$.

It follows from the lemma above that $\operatorname{SAut}\left(F_{\infty}\right)$ is the commutator subgroup of $\operatorname{Aut}\left(F_{\infty}\right)$, which is labelled $E_{\infty}$ in [35].

Under the embedding of $\Sigma_{n}$ in $\operatorname{Aut}\left(F_{n}\right)$, the sign of the permutation corresponds to the determinant of the image of the automorphism in $\mathrm{GL}_{n}(\mathbb{Z})$. Therefore the alternating group $A_{n}$ is correspondingly embedded in $\operatorname{SAut}\left(F_{n}\right)$.

Lemma 2.2. For $n \geq 3, A_{n}$ normally generates $\operatorname{SAut}\left(F_{n}\right)$.

Proof. We use the fact (e.g. [10]) that $\operatorname{SAut}\left(F_{n}\right)$ is normally generated by the left Nielsen automorphisms $\lambda_{i j}$, where $\lambda_{i j}$ sends the $i$ th generator $a_{i}$ of $F_{n}$ to $a_{i} a_{j}$ while fixing all other generators. Let $N$ denote the normal closure of $A_{n}$ in $\operatorname{SAut}\left(F_{n}\right)$. Arguing as in the proof of [8], Proposition 1, we note that the 3-cycle $\left(\begin{array}{lll}i & k\end{array}\right)$ conjugates $\lambda_{i j}$ to $\lambda_{j k}$. Thus, in $\operatorname{SAut}\left(F_{n}\right) / N$ both $\lambda_{i j}$ and $\lambda_{j k}$ have the same image. Consequently, the image of

$$
\lambda_{i k}=\left[\lambda_{i j}, \lambda_{j k}\right]
$$

is trivial. Hence $N=\operatorname{SAut}\left(F_{n}\right)$.

Lemma 2.3. If a group $G$ is the normal closure of a strongly $m$-torsion generated subgroup $H$, then $G$ is also strongly m-torsion generated.

Proof. Evidently, if $H$ is normally generated by an element $x_{m}$ of order $m$, then so is $G$.

Since, from its simplicity for $n \geq 5, A_{n}$ is strongly $\prod m_{i}$-torsion generated whenever $\sum m_{i} \leq n$ and $\operatorname{gcd}\left(m_{i}\right)=1$, it follows from the lemmas above that $\operatorname{SAut}\left(F_{n}\right)$ is too. This gives the first of the following conclusions. 
Theorem 2.4. The groups $\operatorname{SAut}\left(F_{\infty}\right)$ and $\operatorname{SOut}\left(F_{\infty}\right)$ are strongly torsion generated.

Proof. As noted in [7], any nontrivial quotient of a strongly torsion generated group must again be strongly torsion generated, when the kernel is torsion-free. Hence $\operatorname{SOut}\left(F_{\infty}\right)$, as the quotient of $\operatorname{SAut}\left(F_{\infty}\right)$ by the free group $F_{\infty}$, is also strongly torsion generated.

Remark 2.5. It is a curious fact that the group $G=\operatorname{SAut}\left(F_{\infty}\right)$ shares with $G=A_{\infty}$ and $G=E(R)$ the striking property that not only is it strongly torsion generated, but the plus-construction applied to its classifying space, $B G^{+}$, is an infinite loop space [28].

\section{Classical linear groups}

The case of the perfect commutator subgroup $E(R)$ of $\mathrm{GL}(R)$ for an associative ring $R$ with unit was discussed in [2]. More generally, so as to embrace symplectic, unitary and orthogonal groups, one can consider a ring $(R, \Lambda)$ with form parameter $\Lambda$ (as, for example, in [21]). Then the natural candidate for a strongly torsion generated group in this context is the perfect commutator subgroup $\operatorname{EU}(R, \Lambda)$ of $U(R, \Lambda)$ [21], (5.4.6).

One way to approach this is by means of generators and relations for $\operatorname{EU}_{2 n}(R, \Lambda)$ [21], (5.3B), in the spirit of [33]. The alternative treatment given here is perhaps more illuminating.

Our aim is to apply the following, slight strengthening of a lemma of [7], as observed in [5].

Lemma 3.1. Let $H$ be a simple group that, for each $n \geq 2$, has a superperfect subgroup $L_{n}$ possessing an element of order $n$. Suppose that $G$ is a group containing $H$ in such a way that the normal closure of $H$ in $G$ is $G$ itself. Then every perfect central extension of $G$ is strongly torsion generated.

To this end we consider, as usual, $H$ as the infinite alternating group $A_{\infty}$, with $A_{n}$ hyperbolically embedded, via $E_{n}(R)$, in $\operatorname{EU}_{2 n}(R, \Lambda)$. (This is well-known not to be the most efficient embedding, but suffices here.) Evidently, the off-diagonal entries of permutation matrices lie in no proper ideal of $R$. Therefore, for no proper ideal $(a, \Gamma)$ of $(R, \Lambda)$ does the normal closure $N$ of $H$ in $G=\operatorname{EU}(R, \Lambda)$ lie in $U(a, \Gamma)$. Accordingly, the Bass Sandwich Theorem for this situation [21], (5.4.10), dictates that $N$ has level $(R, \Lambda)$, in other words that

$$
\mathrm{EU}(R, \Lambda) \leq N \leq U(R, \Lambda)
$$


Of course, because $N$ lies in $\mathrm{EU}(R, \Lambda)$, we conclude that $N=\mathrm{EU}(R, \Lambda)$, and have the following result.

Theorem 3.2. For every form ring $(R, \Lambda)$, every perfect central extension of the perfect commutator subgroup $\mathrm{EU}(R, \Lambda)$ of $U(R, \Lambda)$ is strongly torsion generated.

Thus in particular, the two extreme central extensions, $\mathrm{EU}(R, \Lambda)$ itself and its universal central extension, the Steinberg group $\operatorname{StU}(R, \Lambda)$, are strongly torsion generated. In the case $R=\mathbb{Z}$, the integral symplectic group $\operatorname{Sp}(\mathbb{Z})$ and the commutator subgroup $O^{\prime}(\mathbb{Z})$ (of index 4) of the integral orthogonal group $O(\mathbb{Z})$ are strongly torsion generated.

\section{Mapping class groups}

Write $S_{g, r}^{s}$ for (a copy of) an oriented smooth surface of genus $g$ with $s$ marked points (often referred to as "punctures") and $r$ boundary components. Thus the boundary $\partial S_{g, r}^{s}$ of $S_{g, r}^{s}$ consists of the disjoint union of $r$ circles, where $r \geq 0$. The (pure) mapping class group $\Gamma_{g, r}^{s}$ is the discrete group of components of the topological group $\operatorname{Diff}_{+}\left(S_{g, r}^{s}\right)$ of orientation-preserving diffeomorphisms of $S_{g, r}^{s}$ that fix the marked points and the boundary pointwise. Conventionally, the suffices $r$ and $s$ are often omitted when zero.

For $r \geq 1$, there are some standard operations that enable one to vary the suffices. For example, to pass from $g$ to $g+1$, glue a two-holed torus $S_{1,2}^{0}$ to $S_{g, r}^{s}$, yielding $S_{g+1, r}^{s}$. Extending diffeomorphisms by the identity produces a homomorphism $\psi: \Gamma_{g, r}^{s} \rightarrow \Gamma_{g+1, r}^{s}$. In this way, we are able to obtain the stable mapping class group $\lim _{g \rightarrow \infty} \Gamma_{g, r}^{s}$, so long as $r \geq 1$ [22]. The restriction $r \geq 1$ here is important for two reasons, as follows.

First, as is well known, each group $\Gamma_{g, r}^{s}$ is torsion-free when $r \geq 1$. It follows that the stable group is also torsion-free, and hence cannot be a candidate for a strongly torsion generated group.

Second, when $r=0$ the group $\Gamma_{g, 0}^{s}$ does indeed contain torsion. For example, it is known that every finite group embeds in some $\Gamma_{g}=\Gamma_{g, 0}^{0}$ [20]. However, the stabilization process described above requires $r>0$. This prompts the question of rigidity (considered for $\operatorname{Aut}\left(F_{m}\right) \rightarrow \operatorname{Aut}\left(F_{n}\right)(m>n)$ in [8], and for $\Gamma_{g} \rightarrow \Gamma_{h}$ $(g>h)$ in [25]): whether for suitable $g, h$ there can exist a nontrivial map from $\Gamma_{g}$ to $\Gamma_{h}$. Of course, with stabilization in mind, the interesting cases have $g<h$. To examine this issue, we begin by reworking a result of Glover and Mislin [12]. (Our statement is easily seen to be equivalent to that of Harvey [24].) In this section, we 
find it convenient to use the notation, for an integer $k$,

$$
\varepsilon_{k}= \begin{cases}1, & k \text { even } \\ 0, & k \text { odd }\end{cases}
$$

Lemma 4.1. $\Gamma_{h}$ contains an element of prime order $2 a+1$ if and only if for some $k \geq 1$

$$
k a \leq h \leq k\left(a+\frac{1}{2}\right)+\varepsilon_{k}
$$

Proof. First, recall that the only odd prime torsion in $\Gamma_{1}=\mathrm{SL}_{2}(\mathbb{Z}) \cong C_{4} * C_{2} C_{6}$ is 3-torsion, so that we may safely assume that $h \geq 2$. Then, [12], (3.3), asserts that $\Gamma_{h}$ contains an element of prime order $2 a+1$ if and only if for some $u \geq 0$ and $v \in\{-2,0,1,2,3, \ldots\}$

$$
h=u(2 a+1)+v a .
$$

We write this last expression as $k a+u$ where $k=2 u+v$. When $k$ is even, this corresponds to all pairs $(u, k-2 u)$ with $u=0, \ldots, \frac{k}{2}+1$; while for $k$ odd we have all pairs $(u, k-2 u)$ with $u=0, \ldots, \frac{k-1}{2}$. The result follows.

It follows immediately from this result that there is a bound on the prime orders of torsion elements, and hence that no $\Gamma_{g}$ can be strongly torsion generated. However, partial strong torsion generation results are usually possible. Throughout, we draw upon the observation that if an element $x$ normally generates a group $G$, then its image $\varphi(x)$ must normally generate any homomorphic image $\varphi(G)$ of $G$. For the case of genus 2, we shall employ the following lemma (doubtless well-known).

Lemma 4.2. For $n \geq 1$, the principal congruence subgroup

$$
\operatorname{Ker}\left[\mathrm{GL}_{n}(\mathbb{Z}) \longrightarrow \mathrm{GL}_{n}\left(\mathbb{F}_{p}\right)\right]
$$

contains no torsion coprime to $p$.

Proof. $I_{n}=A^{k}=\left(I_{n}+p^{v} M\right)^{k}$, with $v$ the maximal $p$-exponent of $A-I_{n}$, yields that

$$
k M=-p^{v} \sum_{i=2}^{k}\left(\begin{array}{l}
k \\
i
\end{array}\right) p^{v(i-2)} M^{i},
$$

which contradicts the choice of $v$ when $p \nmid k$.

Theorem 4.3. (a) If both $g \geq 3$ and $d$ divides $g$ or $g-1$ or $4 g+2$, then $\Gamma_{g}$ is strongly d-torsion generated. 
(b) Conversely, if $\Gamma_{g}$ is strongly $d$-torsion generated, then both $g \geq 3$ and $d \leq$ $4 g+2$, and the prime divisors $p$ of $d$ satisfy

$$
-\frac{2 \varepsilon_{k}}{k} \leq p-\frac{2 g}{k} \leq 1
$$

for some integer $k$.

Proof. We first exclude the cases of low genus. $\Gamma_{0}$ is trivial. For the cases $g=1,2$, we recall that the greatest order of any torsion element of $\Gamma_{g}$ is precisely $4 g+2$. However, $\Gamma_{1} \cong \mathrm{SL}_{2}(\mathbb{Z}) \cong C_{4} *_{C_{2}} C_{6}$ has abelianization cyclic of order 12 , so that it cannot be normally generated by an element of smaller order.

The case of $\Gamma_{2}$ is more subtle, and indeed is the subject of [29], where Mumford shows that $\left(\Gamma_{2}\right)_{\mathrm{ab}}$ is cyclic of order 10 (whence $d=10$ is the only possibility), and that $\Gamma_{2}$ is normally generated by a single, torsion-free element. [25] identifies an element of order 10 in $\Gamma_{2}$ that maps to the fourth power of Mumford's generator of $\left(\Gamma_{2}\right)_{\mathrm{ab}}$, and therefore normally generates an index 2 subgroup of $\Gamma_{2}$.

To contradict the supposition that $\Gamma_{2}$ is normally generated by an element $\varepsilon$ of order 10 , we consider the canonical epimorphisms

$$
\Gamma_{2} \stackrel{\eta}{\longrightarrow} \mathrm{Sp}_{4}(\mathbb{Z}) \stackrel{\pi}{\longrightarrow} \mathrm{Sp}_{4}\left(\mathbb{F}_{2}\right),
$$

and note that each of these groups must be normally generated by the image of $\varepsilon$. Now $\mathrm{Sp}_{4}\left(\mathbb{F}_{2}\right) \cong S_{6}$, and by consideration of cycle lengths one sees that $S_{6}$ contains no element of order 10. Therefore the image of $\varepsilon$ has order 2 or 5 . Now order 5 is not possible, since any element of that order in $S_{6}$ must be an even permutation, with normal closure $A_{6}$. Thus, $\pi \eta(\varepsilon)$ is an involution. Hence, in $\mathrm{Sp}_{4}(\mathbb{Z}) \eta(\varepsilon)$ has square lying in $\operatorname{Ker} \pi$. Because the kernel of $\eta$ is the Torelli group, which is known to be torsion-free, $\eta\left(\varepsilon^{2}\right)$ is an element of order 5 in the congruence subgroup Ker $\pi$. Such a possibility is denied by the lemma above.

When $g \geq 3$, the first assertion is a restatement of Theorems 4, 11 of [25] in our language. (Note that the case $d=2$ follows from Theorem 11 rather than from Theorem 4.)

The converse claim uses our low-genus discussion. The condition $d \leq 4 g+2$ is due to [23], while the inequalities for prime divisors follow from Lemma 4.1 above.

Since for $g \geq 3$ this result affords distinct prime orders of elements that normally generate $\Gamma_{g}$, and whose images therefore generate $\left(\Gamma_{g}\right)_{\mathrm{ab}}$, an elementary consequence is the famous result of [31] that $\Gamma_{g}$ is a perfect group. However, that result is already used in the reasoning of [25] cited in our argument above. The first cases left open by the theorem above are whether $\Gamma_{3}$ is strongly 6-torsion generated, and (for $d$ prime) whether $\Gamma_{5}$ is strongly 3 -torsion generated. 
Theorem 4.4. Consider the set of homomorphisms $\Gamma_{g} \rightarrow \Gamma_{g+m}$ where $g \in \mathbb{N}$, $m \in \mathbb{Z}$.

(a) [25] If $m<0$, then there is only the trivial homomorphism.

(b) If $m=0$ or $g \leq 2$, then there are nontrivial homomorphisms.

(c) Suppose that $g \geq 3, m \geq 1$. Then the only homomorphism $\Gamma_{g} \rightarrow \Gamma_{g+m}$ is the trivial homomorphism, provided that at least one of the following holds.

(i) $g-1=$ pu with prime $p>2(m+u+2)$;

(ii) $g=p v$ with prime $p>2(m+v+1)$, and $m \geq 2$;

(iii) $2 g+1=p w$ with prime $p>m+w+1$.

Proof. (a) is the rigidity result of Harvey and Korkmaz [25], Theorem 7. For (b), when $m=0$ the identity homomorphism is always available, while both $\Gamma_{1}$ and $\Gamma_{2}$ have quotients of order 2 . Since for all $h \geq 1, \Gamma_{h}$ contains an involution, there are always nontrivial homomorphisms $\Gamma_{g} \rightarrow \Gamma_{h}$ when the domain is $\Gamma_{1}$ or $\Gamma_{2}$.

To obtain (c), we apply Theorem 4.3 above. Here is the argument in case (i) (with (ii) and (iii) similar). By (4.3) (a), $\Gamma_{g}$ is strongly $p$-torsion generated. It therefore suffices to use (4.3) (b) to show that $\Gamma_{g+m}$ contains no element of order $p$. This means contradicting the possibility that for some integer $k$ we have

$$
-\frac{2 \varepsilon_{k}}{k} \leq p-\frac{2(g+m)}{k} \leq 1
$$

On multiplying by $k$, substituting $g-1=p u$, and adding 2 throughout, we can rewrite this as

$$
2-2 \varepsilon_{k} \leq(k-2 u) p-2 m \leq k+2 .
$$

The left-hand inequality gives $k \geq 2 u+1$. In conjunction with the right-hand inequality, this yields that

$$
p-1 \leq(k-2 u)(p-1)=(k-2 u) p-k+2 u \leq 2 m+2+2 u,
$$

in defiance of our further hypothesis.

Evidently, for a given positive $m$, the theorem shows that there are infinitely many values of $g$ forcing triviality of $\Gamma_{g} \rightarrow \Gamma_{g+m}$. This prompts the following conjecture.

Conjecture 4.5. Given a positive integer $m$, then for all sufficiently large $g$ the only homomorphism $\Gamma_{g} \rightarrow \Gamma_{g+m}$ is the trivial homomorphism.

From the previous theorem, we may deduce a number-theoretic condition that is sufficient for affirmation. 
Corollary 4.6. Let $m \geq 1$, and let $p$ be the largest prime factor of $g(g-1)(2 g+1)$. If

$$
p-\sqrt{2 g}>2 m+2
$$

(and $m \geq 2$ if $p \mid g$ ), then the only homomorphism $\Gamma_{g} \rightarrow \Gamma_{g+m}$ is the trivial homomorphism.

Proof. Again we give details only for the case where $g-1=p u$, the other two possibilities being similar. From the inequality above,

$$
(p-(2 m+2))^{2}>(\sqrt{2 g})^{2}=2 p u+2 ;
$$

so that

$$
\begin{aligned}
(p-(2 m+2+u))^{2} & >2+2 u(2 m+2)+u^{2} \\
& >(u+2)^{2},
\end{aligned}
$$

which implies that $p>2(m+u+2)$ and thus we are in case (i) of the previous theorem.

We therefore turn the following question over to the number-theorists...

Question 4.7. Let $A(n)$ denote the largest prime factor of $n(n-1)(2 n+1)$, and let $B(n)=A(n)-\sqrt{2 n}$. Given $k>0$, what is the density of the set $\{n \in \mathbb{N} \mid B(n)<k\}$ ?

In view of the preceding corollary, were this set finite, it would imply Conjecture 4.5. However, the following argument shows that such is not the case.

Proposition 4.8. For any $k \in \mathbb{R}$, the set $\{n \in \mathbb{N} \mid B(n)<k\}$ is infinite.

Proof. For $r \geq 0$, define

$$
\left[\begin{array}{l}
u_{r} \\
v_{r}
\end{array}\right]=\left[\begin{array}{ll}
5 & 4 \\
6 & 5
\end{array}\right]^{r}\left[\begin{array}{l}
1 \\
1
\end{array}\right] .
$$

Since $\left[\begin{array}{ll}5 & 4 \\ 6 & 5\end{array}\right]$ commutes with $\left[\begin{array}{ll}0 & 2 \\ 3 & 0\end{array}\right]$, we have

$$
\left[\begin{array}{ll}
u_{r} & 2 v_{r} \\
v_{r} & 3 u_{r}
\end{array}\right]=\left[\begin{array}{ll}
5 & 4 \\
6 & 5
\end{array}\right]^{r}\left[\begin{array}{ll}
1 & 2 \\
1 & 3
\end{array}\right] \in \operatorname{SL}_{2}(\mathbb{Z}) .
$$

(In fact, $\left(2 v_{r}, u_{r}\right)$ is the $2 r$ th convergent in the continued fraction representation for $\sqrt{6}$.) Then $A\left(v_{r}^{2}\right)$ is the largest prime factor of $v_{r}^{2}\left(v_{r}-1\right)\left(v_{r}+1\right) 3 u_{r}^{2}$ and so bounded above by $v_{r}+1$. This leaves

$$
B\left(v_{r}^{2}\right) \leq 1-v_{r}(\sqrt{2}-1) \rightarrow-\infty \quad \text { as } r \rightarrow \infty .
$$


This assertion also follows from [1]. Moreover, the conjecture (1.4) of [27] indicates that the likely answer to Question 4.7 is $(1-\log 2)^{3}=0.0288928 \ldots$.

The contrast with the situation for mapping class groups of surfaces with boundary is stark, as follows.

Lemma 4.9. For $g \geq 2$ and $r=1$, the homomorphism $\psi: \Gamma_{g, r} \rightarrow \Gamma_{g+1, r}$ has $\Gamma_{g+1, r}$ as the normal closure of the image of $\psi$.

Proof. The result follows readily from the presentation of $\Gamma_{g, r}$ found in [36], Theorem $1^{\prime}$. Specifically, all generators there are mapped under $\psi$ to their namesakes in $\Gamma_{g+1, r}$, whose presentation contains the further generators $e_{g}, a_{g+1}$. They commute with all other generators except for the braid relations

$$
a_{g} e_{g} a_{g}=e_{g} a_{g} e_{g}, \quad a_{g+1} e_{g} a_{g+1}=e_{g} a_{g+1} e_{g} .
$$

The former shows that $e_{g}$ lies in the normal closure of $a_{g}$ and so of $\operatorname{Im} \psi$, while the latter in turn forces $a_{g+1}$ also to lie in the normal closure.

Note that we have deliberately phrased the assertion of the lemma above so as to provoke the question as to whether it is also valid when $r>1$.

These results enable us to show that the obvious candidate for stabilization also fails for the subgroup $K_{g}$ of $\Gamma_{g, 1}$ comprising the kernel of the capping homomorphism $\Gamma_{g, 1} \rightarrow \Gamma_{g}$.

Lemma 4.10. Given a map of group extensions

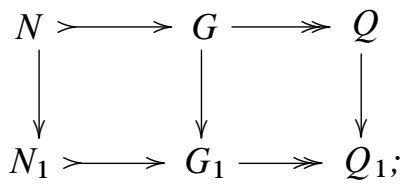

if the image of $G$ in $G_{1}$ normally generates $G_{1}$, then the image of $Q$ in $Q_{1}$ normally generates $Q_{1}$. The converse holds provided also that the image of $N$ in $N_{1}$ normally generates $N_{1}$.

Now, suppose that $\psi: \Gamma_{g, 1} \rightarrow \Gamma_{g+1,1}$ were to restrict to a homomorphism $\psi \mid$ from $K_{g}$ to $K_{g+1}$. Consequently, $\psi$ would induce a homomorphism

$$
\bar{\psi}: \Gamma_{g} \cong \Gamma_{g, 1} / K_{g} \longrightarrow \Gamma_{g+1}
$$

making commutative the diagram

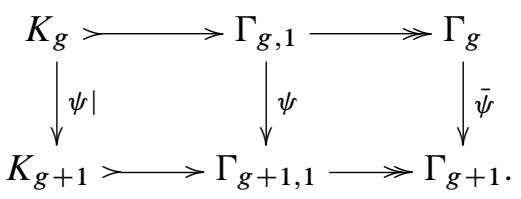


Now suppose that $g \geq 2$. Then, by the last lemma, the image of $\psi$ cannot lie in $K_{g+1}$. Thus, $\bar{\psi}$ is nontrivial. However, under the further assumption that $2 g+1$ is a prime at least 7 , this is contradicted by the theorem above. We conclude that there are infinitely many values of $g$ for which a restriction $\psi \mid: K_{g} \rightarrow K_{g+1}$ fails to exist.

\section{Braid groups}

The braid groups of interest here are of course those that contain some torsion, namely, after [34], those of the 2-sphere and the real projective plane. Presentations of these groups are displayed in [30].

Sphere. The $n$-braid group $B_{n}\left(S^{2}\right)=\Gamma_{0,0}^{n}$ is a quotient of the classical Artin braid group, of braids on the disc. It is well-known to be torsion-generated, and in fact generated by just two torsion elements [14], namely $\alpha_{0}, \alpha_{1}$ as defined in Murasugi's theorem ((b) below). (Here, the cycle type of a braid refers to that of its corresponding induced permutation of its nodes, and $\sigma_{i}$ is the usual generator passing the $i$ th strand over the $(i+1)$ st.)

Theorem 5.1. (a) [9] $B_{n}\left(S^{2}\right)$ has the presentation with generators $\sigma_{i}(1 \leq i \leq n-1)$ and relations

$$
\begin{aligned}
\sigma_{i} \sigma_{j} & =\sigma_{j} \sigma_{i}, & & 1<i+1<j<n, \\
\sigma_{i} \sigma_{i+1} \sigma_{i} & =\sigma_{i+1} \sigma_{i} \sigma_{i+1}, & & 1 \leq i \leq n-2, \\
\sigma_{1} \ldots \sigma_{n-2} \sigma_{n-1}^{2} \sigma_{n-2} \ldots \sigma_{1} & =1 . & &
\end{aligned}
$$

(b) [30] For $n \geq 3$, each torsion element of $B_{n}\left(S^{2}\right)$ is conjugate to a power of one of the following:

- $\alpha_{0}=\sigma_{1} \ldots \sigma_{n-1}$ of order $2 n$, with cycle type $(n)$;

- $\alpha_{1}=\sigma_{1} \ldots \sigma_{n-2} \sigma_{n-1}^{2}$ of order $2(n-1)$, with cycle type $(n-1,1)$;

- $\alpha_{2}=\sigma_{1} \ldots \sigma_{n-3} \sigma_{n-2}^{2}$ of order $2(n-2)$, with cycle type $(n-2,1,1)$.

A simple, no doubt known, consequence is the following, reminiscent of Theorem 4.4 above.

Corollary 5.2. Let $n \geq 4$.

(a) Adjoining a free strand does not induce a homomorphism $B_{n}\left(S^{2}\right) \rightarrow B_{n+1}\left(S^{2}\right)$.

(b) Doubling a strand does not induce a homomorphism $B_{n}\left(S^{2}\right) \rightarrow B_{n+1}\left(S^{2}\right)$. 
Proof. (a) By the above result, if such a homomorphism existed, then it would send $\alpha_{2}$ to an element of finite order having cycle type $(n-2,1,1,1)$. However, by the theorem again (or (4.8) in [11]), any element with such a cycle type must have infinite order.

(b) Similarly, there is some conjugate of $\alpha_{2}$, necessarily of the same cycle type $(n-2,1,1)$, such that the doubled strand is one of the two that is stable under the associated permutation. In that case, we again have the contradiction that its image has finite order dividing $2(n-2)$, but is of cycle type $(n-2,1,1,1)$ and so of infinite order in $B_{n+1}\left(S^{2}\right)$. (For a fuller discussion of the interaction between doubling, deleting and permutations, see [3].)

Thus, as with mapping class groups, the motto is that torsion prohibits stabilization. On the other hand, at the non-stable level we can salvage the following information. For this result and its corollary, we use the obvious fact that in $B_{n}\left(S^{2}\right)$

$$
\sigma_{n-1}=\alpha_{0}^{-1} \alpha_{1}
$$

and, from the defining relator $\sigma_{1} \ldots \sigma_{n-2} \sigma_{n-1}^{2} \sigma_{n-2} \ldots \sigma_{1}$,

$$
\alpha_{0}=\sigma_{1}^{-1} \ldots \sigma_{n-1}^{-1} \text {. }
$$

A quick induction then shows that, for all $i$ with $2 \leq i \leq n-1$,

$$
\sigma_{i-1}=\alpha_{0}^{-1} \sigma_{i} \alpha_{0}
$$

Proposition 5.3. The normal closure of $\alpha_{1}$ in $B_{n}\left(S^{2}\right)$ has index $\operatorname{gcd}(n, 2)$. In particular, for $n$ odd, $B_{n}\left(S^{2}\right)$ is strongly $2(n-1)$-torsion generated.

Proof. Write $\bar{\sigma}_{i}$ to indicate the image of $\sigma_{i}$ in the quotient of $B_{n}\left(S^{2}\right)$ by the normal closure of $\alpha_{1}$. From the relations above, every $\bar{\sigma}_{i}=\alpha_{0}^{-1}$. On the one hand, this makes the quotient group cyclic (generated by $\left.\alpha_{0}\right)$, and thus a quotient of $\left(B_{n}\left(S^{2}\right)\right)_{\mathrm{ab}} \cong$ $C_{2(n-1)}$ (the isomorphism being evident from the defining relations for $B_{n}\left(S^{2}\right)$ ). On the other, the defining expression for $\alpha_{0}$ reduces to $\alpha_{0}=\alpha_{0}^{-(n-1)}$. The result follows.

This leads to another counterpart of the rigidity result Theorem 4.4. Note that the permutation associated to any $n$-braid gives rise to the nontrivial homomorphism, for any $r$,

$$
B_{n}\left(S^{2}\right) \longrightarrow \Sigma_{n} \longrightarrow C_{2} \stackrel{\cong}{\longrightarrow}\left(B_{n+r}\left(S^{2}\right)\right) \longleftrightarrow B_{n+r}\left(S^{2}\right)
$$

In certain circumstances, this is the unique nontrivial homomorphism from $B_{n}\left(S^{2}\right)$ to $B_{n+r}\left(S^{2}\right)$. Here is an example. 
Corollary 5.4. Let $k, r$ be integers with $k, k+r \geq 1$, and suppose that $k$ is coprime to each of $r-1, r$ and $r+1$ (see Remark below). Then the image of any homomorphism $B_{k+1}\left(S^{2}\right) \rightarrow B_{k+r+1}\left(S^{2}\right)$ lies in $\mathcal{Z}\left(B_{k+r+1}\left(S^{2}\right)\right) \cong C_{2}$.

Remark. To have $k$ coprime to $r$ and $r \pm 1$, a necessary condition is that $k \equiv$ $\pm 1(\bmod 6)$; while a sufficient condition is that also $r \equiv \pm 2$ or $\pm 3(\bmod k)$.

Proof. Since the only torsion in $B_{m}\left(S^{2}\right)$ divides $2 m, 2(m-1)$ or $2(m-2)$ [11], the order of the image $\bar{\alpha}_{1}$ of $\alpha_{1}$ is at most 2 . That forces $\bar{\alpha}_{1}$ to lie in $Z\left(B_{k+r+1}\left(S^{2}\right)\right)$ (the unique subgroup of order 2 [11]). Since $\alpha_{0}=\alpha_{1} \sigma_{k}$, we obtain by induction from equation (*) that, for all $i$ with $2 \leq i \leq k, \bar{\sigma}_{i}=\bar{\sigma}_{k}$. Again, the fact that the image is thereby cyclic makes it a quotient of $C_{2 k}$. The defining expression for $\alpha_{1}$ now yields that $\bar{\alpha}_{1}=\bar{\sigma}_{1}^{k+1}$, so that $\bar{\sigma}_{1}^{2(k+1)}=1$. Combining these two facts gives $\bar{\sigma}_{1}^{2}=1$; and hence the image of $B_{k+1}\left(S^{2}\right)$ is of order at most 2 .

Projective plane. As one might expect, despite similarities with the spherical case, now there is a complication according to parity. We thus find it convenient to write $n^{\prime}$ for the greatest odd integer not exceeding $n$. Here is a compilation of the facts that we use.

Theorem 5.5. Let $n \geq 2$.

(a) [30] $B_{n}\left(P^{2}\right)$ has the presentation with generators $\sigma_{i}, \rho_{i}(1 \leq i \leq n-1)$ and $\rho_{n}$, and relations

$$
\begin{aligned}
\sigma_{i} \sigma_{j} & =\sigma_{j} \sigma_{i}, & & 1<i+1<j<n, \\
\sigma_{i} \sigma_{i+1} \sigma_{i} & =\sigma_{i+1} \sigma_{i} \sigma_{i+1}, & & 1 \leq i \leq n-2, \\
\sigma_{i} \rho_{j} & =\rho_{j} \sigma_{i}, & & 1<i+1<j \leq n \text { or } 1 \leq j<i<n, \\
\rho_{i} & =\sigma_{i} \rho_{i+1} \sigma_{i}, & & 1 \leq i \leq n-1, \\
\rho_{i+1}^{-1} \rho_{i}^{-1} \rho_{i+1} \rho_{i} & =\sigma_{i}^{2}, & & 1 \leq i \leq n-1, \\
\sigma_{1} \ldots \sigma_{n-2} \sigma_{n-1}^{2} \sigma_{n-2} \ldots \sigma_{1} & =\rho_{1}^{2} . & &
\end{aligned}
$$

(b) (i) [30], [13] The element

$$
\alpha_{n}= \begin{cases}\sigma_{1} \ldots \sigma_{n-1}, & n \text { odd } \\ \sigma_{1} \ldots \sigma_{n-1} \sigma_{1}, & n \text { even }\end{cases}
$$

has order $2 n^{\prime}$.

(ii) [13] The centre $\mathcal{Z}\left(B_{n}\left(P^{2}\right)\right)$ is generated by the unique element $\left(\alpha_{n}\right)^{n^{\prime}}$ of $B_{n}\left(P^{2}\right)$ of order 2 . 
(iii) [13] A natural number $k$ is the order of an element of $B_{n}\left(P^{2}\right)$ if and only if $k \mid 4 n$ or $k \mid 4(n-1)$.

(c) [18] The commutator subgroup of $B_{n}\left(P^{2}\right)$ is the normal closure in $B_{n}\left(P^{2}\right)$ of $\alpha_{n}$, and $B_{n}\left(P^{2}\right)_{\mathrm{ab}} \cong C_{2} \times C_{2}$.

Although we do not claim that $B_{n}\left(P^{2}\right)$ is strongly $k$-torsion generated for any value of $k$, nevertheless the above information suffices to establish the following rigidity result, comparable to (5.4) above.

Proposition 5.6. For $n \geq 3$, the only nontrivial homomorphism from $B_{n}\left(P^{2}\right)$ to $B_{m}\left(P^{2}\right)$ is

$$
B_{n}\left(P^{2}\right) \longrightarrow \Sigma_{n} \longrightarrow C_{2} \stackrel{\cong}{\longrightarrow} Z\left(B_{m}\left(P^{2}\right)\right) \hookrightarrow B_{m}\left(P^{2}\right)
$$

provided $\operatorname{gcd}\left(n^{\prime}, m\right)=\operatorname{gcd}\left(n^{\prime}, m-1\right)=1$.

Proof. The numerical conditions guarantee that, from (b) in the previous result, $\alpha_{n}$ must have image of order at most 2 , and so be central in $B_{m}\left(P^{2}\right)$. We now use the relations of (a) above. Because the images of $\sigma_{1}$ and $\alpha_{n}$ commute, while $\sigma_{1}$ commutes with all $\sigma_{i}$ with $i \neq 2$, it follows that the images of $\sigma_{1}$ and $\sigma_{2}$ commute. The braid relation between $\sigma_{1}$ and $\sigma_{2}$ then forces that $\bar{\sigma}_{1}=\bar{\sigma}_{2}$ (again using the overline to denote images), and thereby

$$
\bar{\sigma}_{1}=\cdots=\bar{\sigma}_{n-1}
$$

Now $\bar{\rho}_{n}$ commutes with $\bar{\sigma}_{1}$ (since $n \geq 3$ ), and therefore with each $\bar{\sigma}_{i}$, and so with $\bar{\sigma}_{n-1} \bar{\rho}_{n} \bar{\sigma}_{n-1}=\bar{\rho}_{n-1}$. Hence,

$$
\bar{\sigma}_{n-1}^{2}=\left[\bar{\rho}_{n}^{-1}, \bar{\rho}_{n-1}^{-1}\right]=1 .
$$

Therefore, $\bar{\alpha}_{n}=1$. So, from (c) above, the image of the homomorphism must be a nontrivial quotient of $C_{2} \times C_{2}$. However, since $B_{m}\left(P^{2}\right)$ contains a unique element of order 2 , the displayed homomorphism is the only possibility.

Observe that the proposition above fails for $n=2$, since the numerical conditions allow the identity map on $B_{2}\left(P^{2}\right)$.

The same argument as in the proof above may be applied to any finite 2-group quotient of $B_{n}\left(P^{2}\right)(n \geq 3)$.

Corollary 5.7. For $n \geq 3$, the only nontrivial finite 2-group quotients of $B_{n}\left(P^{2}\right)$ are $C_{2}$ and $C_{2} \times C_{2}$. 
Proof. First, observe that if the quotient fails to be abelian, and so a quotient of $B_{n}\left(P^{2}\right)_{\mathrm{ab}} \cong C_{2} \times C_{2}$, then the image $\bar{\alpha}_{n}$ of $\alpha_{n}$ has order 2 . Therefore, $\bar{\alpha}_{n}=\left(\bar{\alpha}_{n}\right)^{n^{\prime}}$, which is the image of the unique element of $B_{n}\left(P^{2}\right)$ of order 2 , and so central in the quotient. However, as in the proof above, this leads to a contradiction.

In contrast, note that $B_{2}\left(P^{2}\right)$ is the generalized quaternion group of order 16 [34], p. 87, and so also stands in contrast to the next two consequences. The results above compare interestingly with recent investigations of Goncalves and Guaschi concerning quaternion subgroups of braid groups [16], [17].

Corollary 5.8. For $n \geq 3$, the only nontrivial nilpotent quotients of $B_{n}\left(P^{2}\right)$ are $C_{2}$ and $C_{2} \times C_{2}$.

Proof. Since $B_{n}\left(P^{2}\right)$ is finitely generated with finite abelianization, any nilpotent quotient must be finite [32], p. 127. Since $B_{n}\left(P^{2}\right)_{\mathrm{ab}} \cong C_{2} \times C_{2}$, such a finite quotient can only be a 2 -group, whence the previous corollary applies.

In particular, there is no nilpotent quotient of class 2 ; so, the following is immediate.

Corollary 5.9. For $n \geq 3$, the lower central series of $B_{n}\left(P^{2}\right)$ terminates at the commutator subgroup $\gamma_{2}\left(B_{n}\left(P^{2}\right)\right)$.

By means of routine computations along the lines of the proof of Theorem 1.4 of [15], one can strengthen this result, for $n \geq 5$, as follows.

Proposition 5.10. For $n \geq 5$, the derived series of $B_{n}\left(P^{2}\right)$ terminates at the commutator subgroup $\left(B_{n}\left(P^{2}\right)\right)^{\prime}$.

The same result was proved for the braid groups of the disc in [19] and of the sphere in [15].

\section{Products of strongly torsion generated groups}

In this section we further enlarge the class of known strongly torsion generated groups by establishing that a finite product of strongly torsion generated groups is strongly torsion generated. We also highlight the problems with attempts to extend to infinite products.

We begin with a more general result. 
Proposition 6.1. Let I be a finite, nonempty indexing set and let $n \geq 2$ be an integer. Suppose given for each $i \in I$ an integer $m_{i} \geq 2$ which is prime to $n$, and a group $G_{i}$ which is strongly $m_{i}$-torsion generated and strongly $n$-torsion generated. Then the cartesian product group $\prod_{i \in I} G_{i}$ is strongly n-torsion generated.

Proof. For each $i$, let $g_{i}, h_{i} \in G_{i}$ be normal generators of order $n$ and $m_{i}$ respectively, and consider the element $g:=\left(g_{i}\right)_{i \in I}$ of the product group $G:=\prod_{i \in I} G_{i}$. Clearly, $g$ is of order $n$ and we intend to show that it is a normal generator of $G$. For the sake of readability, we consider each $G_{i}$ as a subgroup of $G$, in the obvious way. Now, we fix $i \in I$ and we claim that

$$
\langle\langle g\rangle\rangle \supseteq\left\{x^{n} \mid x \in G_{i}\right\} .
$$

Fix an element $x \in G_{i}$. Since $g_{i}$ is a normal generator of $G_{i}$, there exist elements $y_{1}, \ldots, y_{s} \in G_{i}$ (for some $s \geq 1$ ) such that

$$
x=y_{1} g_{i} y_{1}^{-1} \ldots y_{s} g_{i} y_{s}^{-1}
$$

in $G_{i}$, hence in $G$. It follows that the element

$$
y_{1} g y_{1}^{-1} \ldots y_{s} g y_{s}^{-1}
$$

of $G$ has $x$ as $i$ th component, and $g_{j}^{s}$ as $j$ th component for every $j \in I$ distinct from $i$. As a consequence, using that $g_{j}^{n}=e$ for each $j$, we get

$$
\langle\langle g\rangle\rangle \ni\left(y_{1} g y_{1}^{-1} \ldots y_{s} g y_{s}^{-1}\right)^{n}=x^{n},
$$

as claimed. We keep $i \in I$ fixed. From this claim, we get that $h_{i}^{n} \in\langle\langle g\rangle\rangle$. Since $n$ is prime to $m_{i}$, it follows from Bézout's Theorem that $\left\langle\left\langle h_{i}^{n}\right\rangle\right\rangle_{G_{i}}=\left\langle\left\langle h_{i}\right\rangle\right\rangle_{G_{i}}$, and therefore,

$$
\langle\langle g\rangle\rangle \supseteq\left\langle\left\langle h_{i}^{n}\right\rangle\right\rangle_{G}=\left\langle\left\langle h_{i}^{n}\right\rangle\right\rangle_{G_{i}}=\left\langle\left\langle h_{i}\right\rangle\right\rangle_{G_{i}}=G_{i} .
$$

Since this holds for every $i \in I$, and $I$ is finite, we infer that $\langle\langle g\rangle\rangle=G$, completing the proof.

The next corollary is an immediate consequence of the proposition.

Corollary 6.2. A finite cartesian product of strongly torsion generated groups is strongly torsion generated.

The above result is applied in [6].

Remark 6.3. If $\left\{G_{i}\right\}_{i \in I}$ is an infinite collection of groups, then, their restricted product $G:=\prod_{i \in I}^{\prime} G_{i}$ is the subgroup of the cartesian product $\prod_{i \in I} G_{i}$ consisting 
of the elements with finite support, i.e. those elements $\left(g_{i}\right)_{i \in I}$ with $g_{i}=e$ for almost all $i \in I$. Note that $G$ is the filtered colimit of the finite products $\prod_{i \in J} G_{i}$, with $J$ running over the finite subsets of $I$. Now, suppose that each $G_{i}$ is nontrivial. Plainly, any finite subset $F$ of $G$ is contained in some finite subproduct $H:=\prod_{i \in J} G_{i}$ of $G$, i.e. with $J \subseteq I$ finite; as a consequence, the normal closure $\langle\langle F\rangle\rangle_{G}$ is also contained in $H$. In particular, $G$ is not finitely normally generated. This shows that an infinite restricted product of strongly torsion generated groups is never strongly torsion generated. Combined with Corollary 6.2, this implies that strongly torsion generated groups are not closed under filtered colimits.

Example 6.4. We finally discuss the (unrestricted) cartesian product of strongly torsion generated groups, in particular the key example of $\prod_{i \in \mathbb{N}} A_{\infty}$. (As revealed in previous sections of this note, one reason for the importance of $A_{\infty}$ as a strongly torsion generated group is that it normally generates a number of other classical groups, and so, by Lemma 2.3, makes them also strongly torsion generated.)

We show that $\prod_{i \in \mathbb{N}} A_{\infty}$ fails to be strongly 2-torsion generated (the argument clearly generalizes). To see this, recall the well-known fact that the involutions in $A_{\infty}$ are precisely the conjugates in $A_{\infty}$ of products of disjoint transpositions, each of which is conjugate in $A_{\infty}$ to some

$$
a_{k}=(12)(34) \ldots(4 k-34 k-2)(4 k-14 k) \in A_{4 k} \subseteq A_{\infty} .
$$

Moreover, no two distinct $a_{k}$ are conjugate; in fact, $a_{m k}$ is the product of $m$ conjugates of $a_{k}$, and cannot be expressed as the product of fewer than $m$ conjugates of $a_{k}$.

Now consider an arbitrary involution $g=\left(g_{i}\right)_{i} \in \prod_{i \in \mathbb{N}} A_{\infty}$, where we may assume that each $g_{i}$ is conjugate to $a_{k_{i}}$ for some sequence $k_{1}, k_{2}, \ldots$ It follows that the element $a_{i k_{i}}$ is the product of at least $i$ conjugates of $g_{i}$; so the length of the product increases unboundedly as $i$ increases. Hence, $\left(a_{i k_{i}}\right)_{i}$ cannot be expressed as a product of a finite number of conjugates of $g$, and so lies outside the normal closure of $g$.

\section{References}

[1] A. Balog and T. D. Wooley, On strings of consecutive integers with no large prime factors. J. Austral. Math. Soc. Ser. A 64 (1998), 266-276. Zbl 0942.11041 MR 1619809

[2] A. J. Berrick, Torsion generators for all abelian groups. J. Algebra 139 (1991), 190-194. Zbl 0745.20031 MR 1106347

[3] A. J. Berrick, F. R. Cohen, Y. L. Wong and J. Wu, Configurations, braids, and homotopy groups. J. Amer. Math. Soc. 19 (2006), 265-326. Zbl 05015435 MR 2188127

[4] A. J. Berrick and M. Karoubi, Hermitian K-theory of the integers. Amer. J. Math. 127 (2005), 785-823. Zbl 1080.19006 MR 2154370 
[5] A. J. Berrick and M. Matthey, Homological realization of prescribed abelian groups via K-theory. Math. Proc. Cambridge Philos. Soc. 142 (2007), 249-258. Zbl 1124.20036 MR 2314599

[6] A. J. Berrick and M. Matthey, Strongly torsion generated groups from $K$-theory of real $C^{*}$-algebras. J. K-Theory 3 (2009), 309-326. Zbl 05555719 MR 2496450

[7] A. J. Berrick and C. F. Miller III, Strongly torsion generated groups. Math. Proc. Cambridge Philos. Soc. 111 (1992), 219-229. Zbl 0762.20017 MR 1142741

[8] M. R. Bridson and K. Vogtmann, Homomorphisms from automorphism groups of free groups. Bull. London Math. Soc. 35 (2003), 785-792. Zbl 1049.20021 MR 1769698

[9] E. Fadell and J. van Buskirk, The braid groups of $E^{2}$ and $S^{2}$. Duke Math. J. 29 (1962), 243-258. Zbl 0122.17804 MR 0141128

[10] S. Gersten, A presentation for the special automorphism group of a free group. J. Pure Appl. Algebra 33 (1984), 269-279. Zbl 0542.20021 MR 0761633

[11] R. Gillette and J. van Buskirk, The word problem and consequences for the braid groups and mapping class groups of the 2-sphere. Trans. Amer. Math. Soc. 131 (1968), 277-296. Zbl 0169.55303 MR 0231894

[12] H. Glover and G. Mislin, Torsion in the mapping class group and its cohomology. J. Pure Appl. Algebra 44 (1987), 177-189. Zbl 0617.57005 MR 0885103

[13] D. L. Gonçalves and J. Guaschi, The braid groups of the projective plane. Algebr. Geom. Topol. 4 (2004), 757-780. Zbl 1072.55013 MR 2100679

[14] D. L. Gonçalves and J. Guaschi, The braid group $B_{n, m}\left(\mathbb{S}^{2}\right)$ and a generalisation of the Fadell-Neuwirth short exact sequence. J. Knot Theory Ramifications 14 (2005), 375-403. Zbl 1072.55013 MR 2149513

[15] D. L. Gonçalves and J. Guaschi, The lower central and derived series of the braid groups of the sphere. Trans. Amer. Math. Soc. 361 (2009), 3375-3399. Zbl 05567261 MR 2491885

[16] D. L. Gonçalves and J. Guaschi, The quaternion group as a subgroup of the sphere braid groups. Bull. London Math. Soc. 39 (2007), 232-234. Zbl 1120.20035 MR 2323453

[17] D. L. Gonçalves and J. Guaschi, Classification of the virtually cyclic subgroups of the pure braid groups of the projective plane. J. Group Theory, to appear; arXiv:0710.5940v1

[18] L. R. González, On the braid groups of $R P^{2}$. M.Sc. thesis, Centro de Investigación y de Estudios Avanzados, Mexico City, 2008.

[19] E. A. Gorin and V. Ja. Lin, Algebraic equations with continuous coefficients and some problems of the algebraic theory of braids. Math. USSR Sbornik 7 (1969), 569-596. Zbl 0211.54905 MR 0251712

[20] L. Greenberg, Maximal Fuchsian groups. Bull. Amer. Math. Soc. 69 (1963), 569-573. Zbl 0115.06701 MR 0148620

[21] A. J. Hahn and O. T. O'Meara, The Classical Groups and K-Theory. Grundlehren Math. Wiss. 291, Springer-Verlag, Berlin 1989. Zbl 0683.20033 MR 1007302

[22] J.L. Harer, Stability of the homology of the mapping class groups of orientable surfaces. Ann. of Math. 121 (1985), 215-249. Zbl 0579.57005 MR 0786348

[23] W. J. Harvey, Cyclic groups of automorphisms of a compact Riemann surface. Quarterly J. Math. (Oxford) Ser. 217 (1966), 86-97. Zbl 0156.08901 MR 0201629 
[24] W. J. Harvey, On branch loci in Teichmüller space. Trans. Amer. Math. Soc. 153 (1971), 387-399. Zbl 0211.10503 MR 0297994

[25] W. Harvey and M. Korkmaz, Homomorphisms from mapping class groups. Bull. London Math. Soc. 37 (2005), 275-284. Zbl 1066.57020 MR 2119027

[26] M. Korkmaz, Generating the surface mapping class group by two elements. Trans. Amer. Math. Soc. 357 (2005), 3299-3310. Zbl 1079.57018 MR 2135748

[27] G. Martin, An asymptotic formula for the number of smooth values of a polynomial. $J$. Number Theory 93 (2002), 108-182. Zbl 1027.11072 MR 1899301

[28] D. McDuff and G. Segal, Homology fibrations and the "group-completion" theorem. Invent. Math. 31 (1976), 279-284. Zbl 0306.55020 MR 0402733

[29] D. Mumford, Abelian quotients of the Teichmüller modular group. J. Anal. Math. 18 (1967), 227-244. Zbl 0173.22903 MR 0219543

[30] K. Murasugi, Seifert fibre spaces and braid groups. Proc. London Math. Soc. 44 (1982), 71-84. Zbl 0489.57003 MR 0642793

[31] J. Powell, Two theorems on the mapping class group of a surface. Proc. Amer. Math. Soc. 68 (1978), 347-350. Zbl 0391.57009 MR 0494115

[32] D. J. S. Robinson, A Course in the Theory of Groups. Grad. Texts in Math. 80, SpringerVerlag, New York 1982. Zbl 0483.20001 MR 0648604

[33] P. Stanek, Concerning a theorem of L. K. Hua and I. Reiner, Proc. Amer. Math. Soc. 14 (1963), 751-753. Zbl 0116.26104 MR 0648604

[34] J. Van Buskirk, Braid groups of compact 2-manifolds with elements of finite order. Trans. Amer. Math. Soc. 122 (1966), 81-97. Zbl 0138.19103 MR 0189013

[35] K. Vogtmann, Automorphisms of free groups and outer space. Geom. Dedicata 94 (2002), 1-31. Zbl 1017.20035 MR 1950871

[36] B. Wajnryb, An elementary approach to the mapping class group of a surface. Geom. Topol. 3 (1999), 405-466. Zbl 0947.57015 MR 1726532

Received December 29, 2007

A. J. Berrick, Department of Mathematics, National University of Singapore,

Singapore 117543, Republic of Singapore

E-mail: berrick@math.nus.edu.sg

M. Matthey, late of: University of Lausanne, IGAT (Institute for Geometry, Algebra and Topology), Bâtiment BCH, EPFL, 1015 Lausanne, Switzerland 